\title{
$k$-Zero Day Safety: Measuring the Security Risk of Networks against Unknown Attacks
}

\author{
Lingyu Wang ${ }^{1}$, Anoop Singhal ${ }^{2}$, Sushil Jajodia ${ }^{3}$, and Steven Noel ${ }^{3}$ \\ 1 Concordia Institute for Information Systems Engineering \\ Concordia University \\ Montreal, QC H3G 1M8, Canada \\ wang@ciise.concordia.ca \\ 2 Computer Security Division \\ National Institute of Standards and Technology \\ Gaithersburg, MD 20899, USA \\ anoop.singhal@nist.gov \\ 3 Center for Secure Information Systems \\ George Mason University \\ Fairfax, VA 22030-4444, USA \\ \{jajodia, snoel\}@gmu.edu
}

\begin{abstract}
The security risk of a network against unknown zero day attacks has been considered as something unmeasurable since software flaws are less predictable than hardware faults and the process of finding such flaws and developing exploits seems to be chaotic [24]. In this paper, we propose a novel security metric, $k$-zero day safety, based on the number of unknown zero day vulnerabilities. That is, the metric counts at least how many unknown vulnerabilities are required for compromising a network asset, regardless of what vulnerabilities those are. We formally define the metric based on a model of relevant network components. We then devise algorithms for computing the metric. Finally, we discuss how to apply the metric for hardening a network.
\end{abstract}

\section{Introduction}

Today's critical infrastructures and enterprises increasingly rely on networked computer systems. Such systems must thus be secured against potential network intrusions. However, before we can improve the security of a network, we must be able to measure it, since you cannot improve what you cannot measure. We need to measure how secure a network currently is, and how secure it would be after introducing new security mechanisms or configuration changes. The lack of such a capability would render network hardening a blind, if not futile, effort.

Emerging efforts on network security metrics (Section 5 will review related work) typically assign numeric scores to vulnerabilities as their relative exploitability or likelihood. The assignment is usually based on known facts about each vulnerability (e.g., whether it requires an authenticated user account). However, such a methodology is no longer applicable when considering 
zero day vulnerabilities about which we have no prior knowledge or experience. In fact, a major criticism of existing efforts on security metrics is that unknown zero day vulnerabilities are unmeasurable [24]. First, the knowledge about a software system itself is not likely to help because unlike hardware faults, software flaws leading to vulnerabilities are known to be much less predictable. Second, modeling adversaries is not feasible either, because the process of finding flaws and developing exploits is believed to be chaotic. Third, existing metrics for known vulnerabilities are not helpful, because they measure the difficulty of exploiting a known vulnerability but not that of finding a zero day vulnerability.

The incapability of measuring unknown zero day vulnerabilities can potentially diminish the value of security mechanisms since an attacker can simply step outside the implementation and do as he pleases [24]. What is the value of a more secure configuration, if it is equally susceptible to zero day attacks? We thus fall into the agnosticism that security is not quantifiable until we can fix all security flaws (by then we certainly do not need any security metric, either).

In this paper, we propose a novel security metric, $k$-zero day safety, based on the number of unknown zero day vulnerabilities. Specifically, instead of attempting to measure which zero day vulnerability is more likely, our metric simply counts how many distinct zero day vulnerabilities a network can resist, regardless of what vulnerabilities those are; a larger number indicates a relatively more secure network, since the likelihood of having more distinct unknown vulnerabilities all available at the same time, applicable to the same network, and exploitable by the same attacker, will be lower. Based on an abstract network model, we formally define the metric and prove it to satisfy the three algebraic properties of a metric function. To compute the metric, we design a series of algorithms and study their complexity. Finally, we demonstrate the power of the metric by applying it to network hardening, and we discuss practical issues in instantiating the model.

The contribution of this work is twofold. First, to the best of our knowledge, this is the first effort capable of quantifying the security risk of a network against unknown zero day attacks. Second, we believe the metric would bring about new opportunities to the evaluation, hardening, and design of secure networks.

In the rest of this paper, we first build intuitions through a running example. We then define the model and metric in Section 2, design and analyze algorithms in Section 3, discuss network hardening and model instantiation in Section 4, review related work in Section 5, and finally conclude the paper in Section 6.

\subsection{Motivating Example}

The left-hand side of Figure 1 shows a toy example where host 1 provides an HTTP service ( $h t t p)$ and a secure shell service $(s s h)$, and host 2 provides only 
$s s h$. The firewall allows traffic to and from host 1, but only connections originated from host 2. Assume the main security concern is over the root privilege on host 2. Clearly, if all the services are free of known vulnerabilities, a vulnerability scanner or attack graph will both lead to the same conclusion, that is, the network is secure (an attacker on host 0 can never obtain the root privilege on host 2), and no additional network hardening effort is necessary.

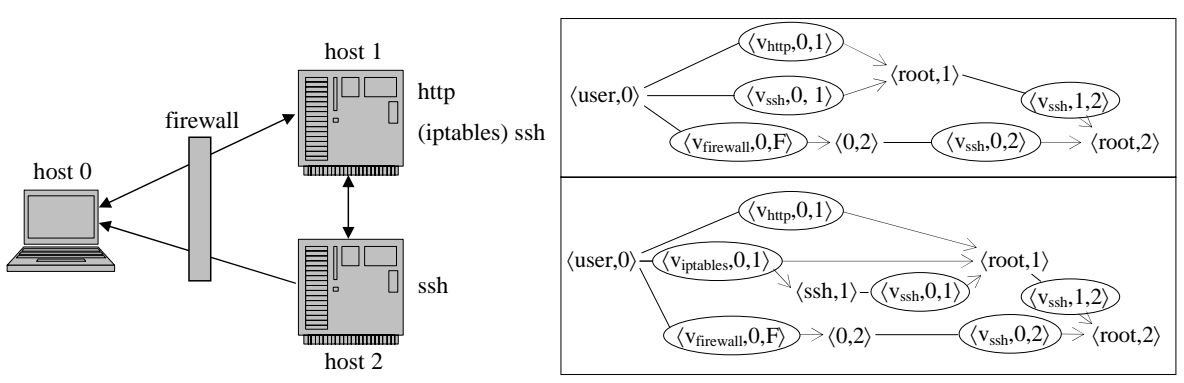

Fig. 1. Network Configuration and Sequences of Zero Day Attacks

However, we shall reach a different conclusion by considering how many distinct zero day attacks the network can resist. The upper-right corner of Figure 1 shows three sequences of zero day attacks leading to $\langle$ root, 2$\rangle$ (each pair denotes a condition and each triple inside oval denotes the exploitation of a zero day vulnerability): An attacker on host 0 can exploit a zero day vulnerability in either http or $s s h$ on host 1 to obtain the root privilege; using host 1 as a stepping stone, he/she can exploit a zero day vulnerability in $s s h$ on host 2 to reach $\langle$ root, 2$\rangle$; alternatively, he/she can exploit a zero day vulnerability in the firewall (e.g., a weak password in its Web-base remote administration interface) to re-establish the blocked connection to host 2 and then exploit $s s h$ on host 2 . The network can resist at most one zero day attack since the second sequence only requires one unique zero day vulnerability in $s s h$ (on both host 1 and 2).

Now consider hardening the network by using iptables rules (iptables) to allow only specific hosts, not including host 0 , to connect to $s s h$ on host 1 . The lower-right corner of Figure 1 shows four sequences of zero day attacks (the two new sequences indicate exploiting a zero day vulnerability in iptables to either connect to $s s h$, or obtain the root privilege, on host 1). It can be observed that all four sequences now require two distinct zero day vulnerabilities. The seemingly unnecessary hardening effort thus allows the network to resist one more zero day attack. The hardened network can thus be considered relatively more secure, since the likelihood of having more zero day vulnerabilities available at the same time, in the same network, and exploitable by the same attacker, will be 
lower ${ }^{1}$. Therefore, the number of distinct zero day vulnerabilities can be used to measure the relative security risk of different networks, which may otherwise be indistinguishable by existing techniques.

On the other hand, the above discussions clearly oversimplify many important issues. For example, we have assumed $s s h$ on host 1 and 2 both correspond to the same zero day vulnerability, which is not necessarily true; similarly, exploiting http and ssh may not necessarily lead to the root privilege; we have not considered known vulnerabilities, which may interact with zero day vulnerabilities; finally, an insider attack may directly give attackers a privilege without any zero day vulnerability. We shall address such issues in the rest of this paper.

\section{Modeling $k$-Zero Day safety}

In this section, we define the $k$-zero day safety metric based on an abstract model of network components.

\subsection{The Network Model}

Definition 1 gives an abstract model of network components relevant to measuring zero day attacks (all notations will be summarized in Table 1). The model will allow us to more formally define and reason about the proposed security metric. Practical issues in instantiating the model will be addressed in Section 4.

Definition 1 (Network). Our network model has the following components:

- H, S, and P, which denotes the set of hosts (computers and networking devices), services, and privileges, respectively.

- $\operatorname{serv}():. H \rightarrow 2^{S}$ and priv(.) $: H \rightarrow 2^{P}$, which denotes a function that maps each host to a set of services and that of privileges, respectively.

- conn $\subseteq H \times H$, which denotes a connectivity relation.

In the model, hosts are meant to also include networking devices because such devices are vulnerable to zero day attacks, and a compromised device may re-enable accesses to blocked services (e.g., the firewall in Figure 1).

A service in the model is either remotely accessible over the network, in which case called a remote service, or used to disable a remote service or network connection, in which case called a security service. The model does not include services or applications that can only be exploited locally for a privilege

\footnotetext{
${ }^{1}$ This likelihood would decrease exponentially in the number of vulnerabilities if such vulnerabilities can be modeled as i.i.d. random variables, but we shall not assume any specific model since the process of developing exploits is believed to be chaotic [24].
} 
escalation (modeling such applications may not be feasible at all considering that an attacker may install his/her own applications after obtaining accesses to a host). On the other hand, the model includes remote services and connectivity currently disabled by security services, since the former may be re-enabled through zero day attacks on the latter (e.g., ssh behind iptables in Figure 1).

In the model, privileges are meant to include those under which services are running and those that can potentially be obtained through a privilege escalation. The purpose of including the latter is not to model privilege escalation itself but to model the strength of isolation techniques (e.g., sandboxing or virtual machines) that may prevent such an escalation, as we shall elaborate shortly.

Example 1. In Figure 1, we have

- $H=\{0,1,2, F\}(F$ denotes the firewall),

- conn $=\{\langle 0, F\rangle,\langle 0,1\rangle,\langle 0,2\rangle,\langle 1, F\rangle,\langle 1,0\rangle,\langle 1,2\rangle,\langle 2, F\rangle,\langle 2,0\rangle,\langle 2,1\rangle\}$ (we include $\langle 0,2\rangle$ since it can be enabled by a zero day attack on the firewall),

$-\operatorname{serv}(1)=\{h t t p$, ssh, iptables $\}, \operatorname{serv}(2)=\{\operatorname{ssh}\}$, and $\operatorname{serv}(F)=\{$ firewall\} (firewall is a security service and it disables connection $\langle 0,2\rangle$ ),

$-\operatorname{priv}(1)=\operatorname{priv}(2)=\{$ user, root $\}$.

\subsection{The Zero Day Attack Model}

The very notion of unknown zero day vulnerability prevents us from assuming vulnerability-specific properties, such as likelihood and severity. We can, however, assume generic properties common to most vulnerabilities, as in Definition 2 (our discussion will be limited to such zero day vulnerabilities).

Definition 2 (Zero Day Vulnerability). A zero day vulnerability is a vulnerability whose details are unknown except that it satisfies the following ${ }^{2}$.

1. It cannot be exploited unless

(a) a network connection exists between the source and destination hosts,

(b) a remote service with the vulnerability exists on the destination host,

(c) and the attacker already has a privilege on the source host.

2. Its exploitation can potentially yield any privilege on the destination host.

The assumptions essentially depict a worst-case scenario about the pre- and post-conditions, respectively, of exploiting a zero day vulnerability. That is, a particular zero day vulnerability may in reality require stronger pre-conditions while implying weaker post-conditions than those stated above. This fact ensures our metric to always yield a conservative result (a future direction is to

\footnotetext{
${ }^{2}$ A zero day vulnerability may refer to a known vulnerability without a fix in other contexts.
} 
extend our work when weaker assumptions can be safely made). For a similar purpose, we shall assign one zero day vulnerability to each service although in reality a service may have more vulnerabilities (note that a more conservative result of a metric is one that requires less zero day vulnerabilities).

We more formally state above assumptions in Definition 3 and 4. In Definition 3, the zero day exploit of a privilege will act as a placeholder when we later model isolation techniques. In Definition 4, unlike the exploit of a known vulnerability which has its unique pre- and post-conditions, all zero day exploits share the same hard-coded conditions, as assumed above. Also note that the zero day exploit of each security service has additional post-conditions, which indicates the exploit will re-enable the disabled conditions. For zero day exploits of a privilege, the pre-conditions include the privilege of every service since we assume that a zero day exploit may potentially yield any privilege.

Definition 3 (Zero Day Exploit). For each $h \in H$ and $x \in(\operatorname{serv}(h) \cup$ priv $(h))$, denote by $v_{x}$ a zero day vulnerability. A zero day exploit is the triple

- $\left\langle v_{s}, h, h^{\prime}\right\rangle$ where $\left\langle h, h^{\prime}\right\rangle \in$ conn and $s \in \operatorname{serv}\left(h^{\prime}\right)$, or

- $\left\langle v_{p}, h, h\right\rangle$ where $p \in \operatorname{priv}(h)$.

Definition 4 (Condition). Denote by $E_{0}$ the set of all zero day exploits, $C_{0}$ the set of conditions (conn $\cup\{\langle x, h\rangle: h \in H, x \in \operatorname{serv}(h) \cup \operatorname{priv}(h)\})$, and define functions pre(.) $: E_{0} \rightarrow C_{0}$ and post(.) $: E_{0} \rightarrow C_{0}$ as

- $\operatorname{pre}\left(\left\langle v_{s}, h, h^{\prime}\right\rangle\right)=\left\{\left\langle h, h^{\prime}\right\rangle,\left\langle s, h^{\prime}\right\rangle,\left\langle p_{\text {min }}, h\right\rangle\right\}$ for each $s \in \operatorname{serv}(h)$, where $p_{\min }$ is the least privilege on $h$.

- pre $\left(\left\langle v_{p}, h, h\right\rangle\right)=\left\{p_{s}: s \in \operatorname{serv}(h), p_{s} \neq p\right\}$ for each $p \in \operatorname{priv}(h)$.

- $\operatorname{post}\left(\left\langle v_{s}, h, h^{\prime}\right\rangle\right)=\left\{p_{s}\right\}$ for each remote service $s$ with privilege $p_{s}$.

- $\operatorname{post}\left(\left\langle v_{s}, h, h^{\prime}\right\rangle\right)=\left\{p_{s}\right\} \cup C_{s}$ for each security service $s$, where $C_{s}$ is the set of conditions disabled by $s$.

- $\operatorname{post}\left(\left\langle v_{p}, h, h\right\rangle\right)=\{\langle p, h\rangle\}$ for each $p \in \operatorname{priv}(h)$.

In Definition 5, a zero day attack graph is composed by relating both exploits of known vulnerabilities and zero day exploits through common pre- and postconditions. In a zero day attack graph, the exploits of known vulnerabilities can be considered as shortcuts that help attackers to satisfy a condition with less zero day exploits. Therefore, exploits of known vulnerabilities here may also be a trust relationship or a misconfigured application, as long as they serve the same purpose of a shortcut for bypassing zero day exploits.

Definition 5 (Zero Day Attack Graph). Given the set of exploits of known vulnerabilities $E_{1}$ and their pre-and post-conditions $C_{1}$, let $E=E_{0} \cup E_{1}$, 
$C=C_{0} \cup C_{1}$, and extend pre(.) and post(.) to $E \rightarrow C$ (as the union of relations). The directed graph $G=\langle E \cup C,\{\langle x, y\rangle:(y \in E \wedge x \in \operatorname{pre}(y)) \vee$ $(x \in E \wedge y \in \operatorname{post}(x))\}\rangle$ is called a zero day attack graph.

In Definition 6, the notion of initial condition serves two purposes. First, it includes all conditions that are not post-conditions of any exploit (which is the usual interpretation of the notion). Second, it is meant to also include conditions that may be satisfied as the result of insider attacks or user mistakes. In another word, the effect of such attacks or mistakes is modeled as the capability of satisfying post-conditions of an exploit without first executing the exploit. Also note that in the definition, an attack sequence is defined as a total order, which means multiple attack sequences may correspond to the same set of partially ordered exploits. However, this is not a limitation since our metric will not require the attack sequence to be unique, as we shall show.

Instead of the usual way of modeling an asset as a single condition, we take a more general approach. The logical connectives $\wedge, \vee$, and $\neg$ respectively model cases where multiple conditions must be satisfied altogether to cause a damage (e.g., the availability of a file with multiple backups on different hosts), cases where satisfying at least one condition will cause the damage (e.g., the confidentiality of the aforementioned file), and cases where conditions are not to be satisfied during an attack (for example, conditions that will trigger an alarm). The asset value is introduced as the relative weight of independent assets.

Definition 6 (Initial Condition, Attack Sequence, and Asset). Given a zero day attack graph $G$,

- the set of initial conditions is given as any $C_{I} \subseteq C$ satisfying $C_{I} \supseteq\{c$ : $(\forall e \in E)(c \notin \operatorname{post}(e))\}$,

- an attack sequence is any sequence of exploits $e_{1}, e_{2}, \ldots, e_{j}$ satisfying $(\forall i \in$ $[1, j])\left(\forall c \in \operatorname{pre}\left(e_{i}\right)\right)\left(c \in C_{I}\right) \vee\left(\exists x \in[1, i-1] c \in \operatorname{post}\left(e_{x}\right)\right)$,

- an asset a is any logical proposition composed of conditions and the logic connectives $\wedge, \vee$, and $\neg$ for which an asset value $v(a)$ is given through a function $v():. A \rightarrow[0, \infty)$ where $A$ denotes the set of all assets, and

- define a function seq(.) $: A \rightarrow 2^{Q}$ as $\operatorname{seq}(a)=\left\{e_{1}, e_{2}, \ldots, e_{j}: a \in\right.$ post $\left.\left(e_{j}\right)\right\}$ where $Q$ denotes the set of all attack sequences.

Example 2. Figure 2 shows the zero day attack graph of our running example,

- if we do not consider insider attacks or user mistakes, the following attack sequences will lead to the asset $\langle$ root, 2$\rangle$.

1. $\left\langle v_{\text {http }}, 0,1\right\rangle,\left\langle v_{\text {ssh }}, 1,2\right\rangle,\left\langle v_{\text {root }}, 2,2\right\rangle$

2. $\left\langle v_{\text {iptables }}, 0,1\right\rangle,\left\langle v_{\text {ssh }}, 1,2\right\rangle,\left\langle v_{\text {root }}, 2,2\right\rangle$ 
3. $\left\langle v_{\text {iptables }}, 0,1\right\rangle,\left\langle v_{\text {ssh }}, 0,1\right\rangle,\left\langle v_{\text {ssh }}, 1,2\right\rangle,\left\langle v_{\text {root }}, 2,2\right\rangle$

4. $\left\langle v_{\text {firewall }}, 0, F\right\rangle,\left\langle v_{\text {ssh }}, 0,2\right\rangle,\left\langle v_{\text {root }}, 2,2\right\rangle$

- if we consider insider attacks on host 1 , only sequence $\left\langle v_{s s h}, 1,2\right\rangle,\left\langle v_{\text {root }}, 2,2\right\rangle$ and the fourth attack sequence above will be needed to compromise $\langle$ root, 2$\rangle$.

- if we consider a different asset $\langle$ root, 1$\rangle \wedge\langle$ root, 2$\rangle$, then only the first three attack sequences above can compromise this asset.

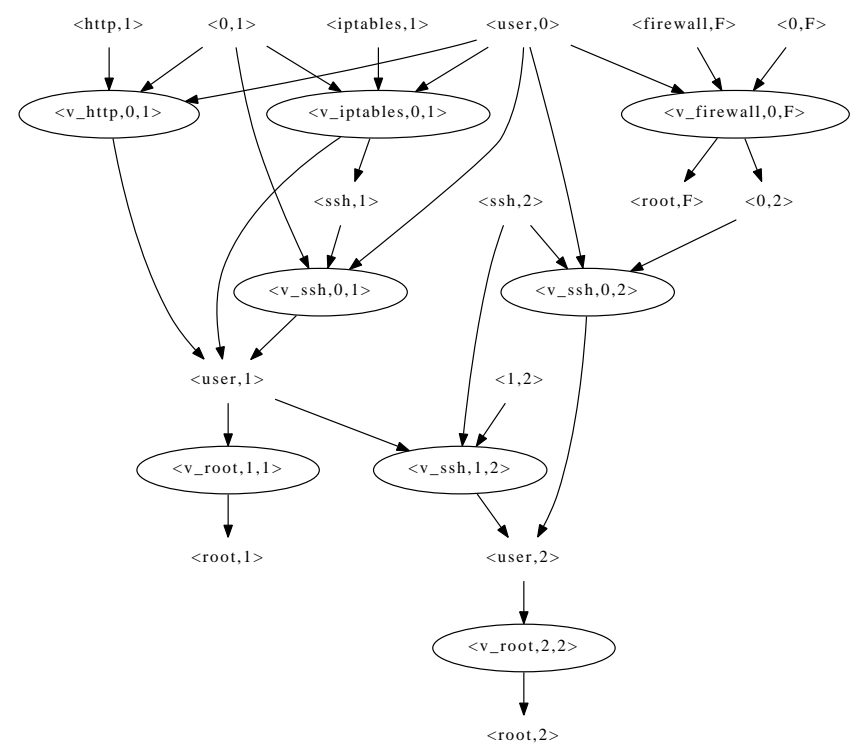

Fig. 2. An Example of Zero Day Attack Graph

\subsection{The $k$-Zero Day Safety Model}

In Definition 7 , the relation $\equiv_{v}$ models two distinct cases in which two zero day exploits should only be counted once. First, both exploits involve the same zero day vulnerability. Second, the exploit of a service is related to the exploit of a privilege to indicate that the former will directly yield the privilege due to the lack of isolation between the two (note that we do not model the details of any involved privilege escalation). A probability can be associated to relation $\equiv_{v}$ to indicate the degree of similarity or isolation, when such information is available. Although the relationship between exploits has distinct meanings in those two cases, the effect of such a relationship towards our metric will be the same. Therefore, the relation $\equiv_{v}$ models such relationships in a unified way.

Given two sets of zero day exploits, the function $k 0 d($.) counts how many exploits in their symmetric difference are distinct (that is, these exploits cannot be related through $\equiv_{v}$ ). In particular, if one of the sets is empty, then the function 
$k 0 d($.$) will yield the number of distinct zero day exploits in the other set. When$ a probabilistic approach is adopted in defining the relation $\equiv_{v}$, the function $k 0 d($.$) can be revised to give the expected value (mean). The reason of defining$ the function over the symmetric difference of two sets is given in Theorem 1.

Definition 7 (Relation $\equiv_{v}$ and Metric Function $k 0 d($.$) ).$

- Define a relation $\equiv_{v} \subseteq E_{0} \times E_{0}$ such that $e \equiv_{v} e^{\prime}$ indicates either e and $e^{\prime}$ are exploits of the same zero day vulnerability, or $e=\left\langle v_{s}, h_{1}, h_{2}\right\rangle, e^{\prime}=$ $\left\langle v_{p}, h_{2}, h_{2}\right\rangle$ and exploiting s yields $p$. We say e and $e^{\prime}$ are distinct if $e \not \equiv v, e^{\prime}$.

- Define a function $k 0 d():. 2^{E_{0}} \times 2^{E_{0}} \rightarrow[0, \infty]$ as $k 0 d\left(F, F^{\prime}\right)=\max \left(\left\{\left|F^{\prime \prime}\right|\right.\right.$ : $\left.\left.F^{\prime \prime} \subseteq\left(F \triangle F^{\prime}\right),\left(\forall e_{1}, e_{2} \in F^{\prime \prime}\right)\left(e_{1} \not \equiv_{v} e_{2}\right)\right\}\right)$ where $\left|F^{\prime \prime}\right|$ denotes the cardinality of $F^{\prime \prime}, \max ($.$) denotes the maximum value in a set, and F \triangle F^{\prime}$ denotes the symmetric difference (that is, $\left(F \backslash F^{\prime}\right) \cup\left(F^{\prime} \backslash F\right)$ ).

Theorem 1. The function $k 0 d($.$) is a metric.$

Proof: See Appendix A.

In Definition 8, we apply the metric $k 0 d($.$) to assets, sets of assets, and a$ network. First, $k 0 d(a)$ indicates the minimum number of distinct zero day exploits required to compromise $a$. This number is unique for each asset, although multiple attack sequences may compromise the asset. The empty set in the definition can be interpreted as the conjunction of all initial conditions (which can always be compromised without any zero day exploit). Second, the metric is applied to a set of independent assets by taking the weighted average with asset values as the weight. Finally, by applying the metric to all assets, we obtain a measurement of a network's resistance to potential zero day attacks.

Definition 8 ( $k$-Zero Day Safety). Given a zero day attack graph $G$, the set of initial conditions $C_{I}$, and the set of assets $A$,

- for any $a \in A$, we use $k 0 d(a)$ for $\min \left(\left\{k 0 d\left(q \cap E_{0}, \phi\right): q \in \operatorname{seq}(a)\right\}\right)$ where min(.) denotes the minimum value in a set and $q$ stands for both a sequence and a set. For any $k \in[0, \operatorname{kod}(a))$, we say a is $k$-zero day safe.

- given any $A^{\prime} \subseteq A$, we use $k 0 d\left(A^{\prime}\right)$ for $\sum_{a \in A^{\prime}}(k 0 d(a) \cdot v(a)) / \sum_{a \in A^{\prime}} v(a)$. For any $k \in\left[0, \operatorname{kod}\left(A^{\prime}\right)\right)$, we say $A^{\prime}$ is $k$-zero day safe.

- in particular, when $A^{\prime}=A$, we say the network is $k$-zero day safe.

Example 3. For the running example, suppose all exploits of services involve distinct vulnerabilities except $\left\langle v_{s s h}, 0,1\right\rangle,\left\langle v_{s s h}, 1,2\right\rangle$, and $\left\langle v_{s s h}, 0,2\right\rangle$. Assume ssh and http are not protected by isolation but iptables is protected. Then, the relation $\equiv_{v}$ is shown in the left-hand side of Table 1 where 1 indicates two exploits are related and 0 the opposite (or, by adopting a probabilistic approach, these can be regarded as the probabilities associated with the relation $\equiv_{v}$ ). 


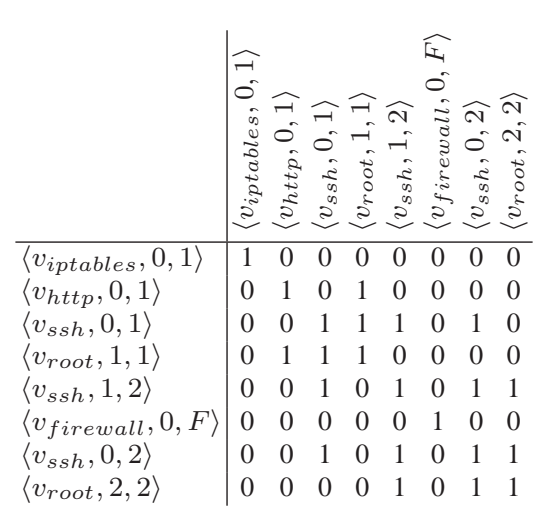

\begin{tabular}{|l|l|}
\hline$H, h$ & A set of hosts, a host \\
$S, s$ & A set of services, a service \\
$P, p$ & A set of privileges, a privilege \\
$\operatorname{serv}()$. & Services on a host \\
$\operatorname{priv}()$. & Privileges on a host \\
$\operatorname{conn}$ & Connectivity \\
$v_{s}, v_{p}$ & Zero day vulnerability \\
$\left\langle v_{x}, h, h^{\prime}\right\rangle$ & Zero day exploit \\
$\left.\operatorname{pre}(),. \operatorname{post}_{(.)}\right)$ & Pre- and post-conditions \\
$G$ & Zero day attack graph \\
$C_{I}$ & Initial conditions \\
$e_{1}, e_{2}, \ldots, e_{j}$ & Attack sequence \\
$A$ & Assets \\
$\operatorname{seq}(a)$ & Attack sequences compromising $a$ \\
$\equiv v$ & Relation of non-distinct exploits \\
$k 0 d()$. & The $k$-zero day safety metric \\
\hline
\end{tabular}

Table 1. An Example of Relation $\equiv_{v}$ (Left) and the Notation Table (Right)

\section{Computing $k$-Zero Day Safety}

This section presents algorithms for computing the $k$-zero day safety.

\subsection{Computing the Value of $k$}

To compute the $k$-zero day safety of a network, we first derive a logic proposition of each asset in terms of exploits. Then, each conjunctive clause in the disjunctive normal form (DNF) of the derived proposition will correspond to a minimal set of exploits that jointly compromise the asset. The value of $k$ can then be decided by applying the metric $k 0 d($.) to each such conjunctive clause.

More precisely, we interpret a given zero day attack graph as a logic program by regarding each exploit or condition as a Boolean variable and by having a logic proposition $c \leftarrow$. for each initial condition $c$, a proposition $e \leftarrow$ $\bigwedge_{c \in \text { pre(e) }} c$ and a set of propositions $\{c \leftarrow e: c \in \operatorname{post}(e)\}$ for each pre- and post-condition relationship, respectively. We can then apply Procedure $k 0 d \_B w d$ shown in Figure 3 to obtain the value of $k$. The main loop (lines 1-8) computes the $k$-zero day safety for each asset. The results of all iterations are aggregated as the final output (line 9). The inner loop (lines 3-6) repetitively applies the afore-mentioned logic propositions to derive a formula, which is converted into its DNF (line 7) from which the $k$-zero day safety is computed (line 8).

Note that a negated condition given in the asset will be replaced with the negation of exploits, whereas the latter will not be further processed (as indicated in line 6). The interpretation is that in order not to satisfy a condition, it suffices not to execute those exploits that have the condition as their postcondition (on the other hand, to satisfy a condition requires more actions). Also note that we have omitted the simplification of logic propositions using logic 


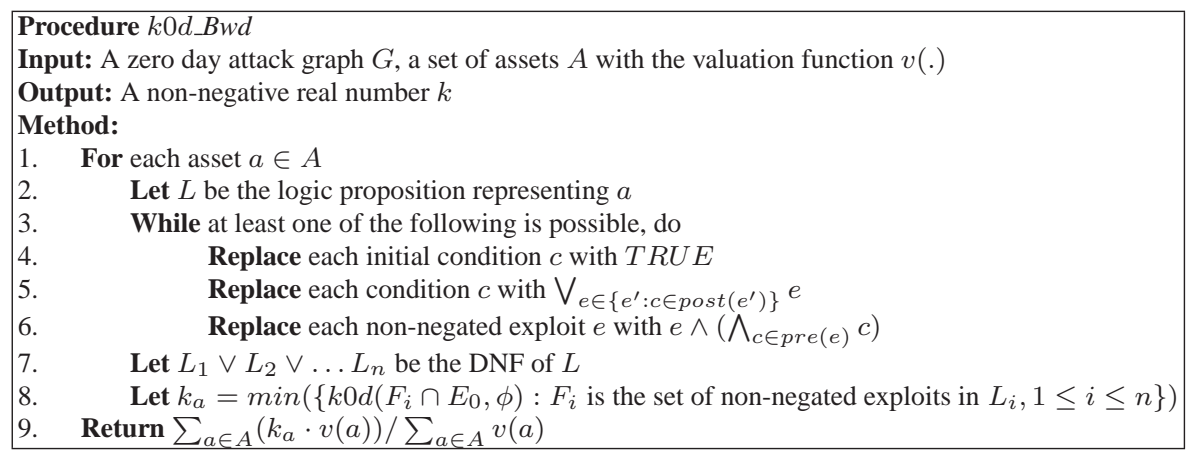

Fig. 3. Computing the Value of $k$

tautologies (such as $e_{1} \wedge \neg e_{1} \equiv F A L S E$ ) and the handling of cycles in the attack graph by maintaining the set of predecessors for each visited node [46].

Complexity The procedure's worst-case complexity is exponential in the size of the zero day attack graph. Specifically, the complexity is dominated by the size of the derived proposition $L$ and its DNF; both may be exponential. Indeed, Theorem 2 shows that the problem of computing $k$-zero day safety is NP-hard.

Theorem 2. Given a zero day attack graph, an asset a, and any non-negative integer $k$, the problem of finding an attack sequence $q \in \operatorname{seq}(a)$ that minimizes $k 0 d\left(q \cap E_{0}, \phi\right)$ is NP-complete.

Proof: See Appendix B.

Note that the intractability result here only implies that a single algorithm is not likely to be found to efficiently determine $k$ for all possible inputs (that is, arbitrary zero day attack graphs). However, efficient solutions still exist for practical purposes. We shall examine two such cases in the following.

\subsection{Determining $k$-Zero Day Safety for a Given Small $k$}

For many practical purposes, it may suffice to know that every asset in a network is $k$-zero day safe for a given value of $k$, even though the network may in reality be $k^{\prime}$-zero day safe for some unknown $k^{\prime}>k$ (note that we have shown determining $k^{\prime}$ to be intractable). We now describe a solution whose complexity is polynomial in the size of a zero day attack graph if $k$ is a constant compared to this size. Roughly speaking, we attempt to compromise each asset with less than $k$ distinct zero day exploits through a forward search of limited depth. The asset is not $k$-zero day safe if any branch of the search succeeds, and vice versa.

Specifically, Figure 4 shows the recursive Procedure $k 0 d \_F w d$ with two base cases (lines 1-2 and 3-4, respectively) and one recursive case (lines 5-9). In 
the first base case, the procedure returns $F A L S E$ when asset $a$ can be compromised with less than $k$ distinct zero day exploits in $T_{e}$. The Sub-Procedure $k 0 d \_$Reachable expands $T_{e}$ with all reachable known exploits since they do not count in terms of the $k 0 d($.$) metric. In the second base case, the procedure re-$ turns $T R U E$ when the set $T_{e}$ already has more than $k$ distinct zero day exploits (regardless of whether $a$ can be satisfied with $T_{c}$ ).

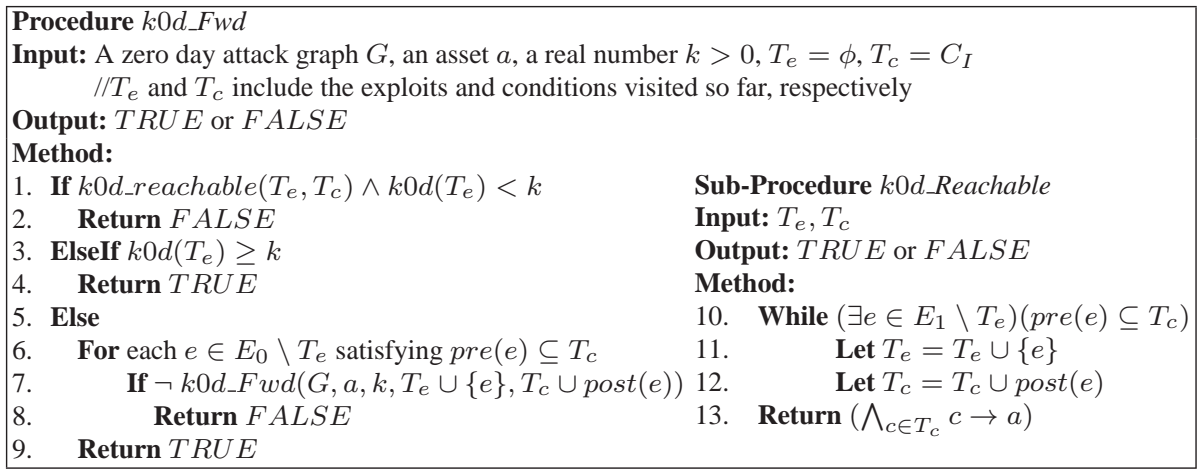

Fig. 4. Determining $k$-Zero Day Safety for a Given $k$

The main procedure enters the recursive case only when $T_{e}$ includes less than $k$ distinct zero day exploits and $a$ cannot be satisfied with $T_{c}$. In this case, the Sub-Procedure $k 0 d$ Reachable must have already added all known exploits and their post-conditions to $T_{e}$ and $T_{c}$, respectively. Now the main procedure iteratively visits each zero day exploit $e$ reachable from $T_{c}$ (line 6), and starts a recursive search from $e$ (line 7). If no such $e$ exists, the procedure will return $T R U E$ indicating the end of a sequence is reached (line 9). If any branch of the search succeeds, $F A L S E$ will be recursively returned to indicate $a$ is not $k$-zero day safe (line 8); otherwise, $T R U E$ is returned (line 9).

Complexity To find reachable known exploits from $E_{1}$, the sub-procedure will check the pre-conditions of each known exploit, which takes time $O\left(|C| \cdot\left|E_{1}\right|\right)$. This will be repeated upon adding an exploit to $T_{e}$ and its post-conditions to $T_{c}$. Therefore, $k 0 d$ Reachable takes time $O\left(|C| \cdot\left|E_{1}\right|^{2}\right)$, which is also the complexity for the base cases of the main procedure since it dominates the complexity of other steps. For the recursive case, we have the recurrence formula $t=O\left(|C| \cdot\left|E_{1}\right|^{2}\right)+\left|E_{0}\right| \cdot t^{\prime}$ where $t$ and $t^{\prime}$ denote the complexity of the recursive case and that of each recursive call. Since the recursive case cannot be entered unless $k 0 d\left(T_{e}\right)<k$ and each recursive call will add one more zero day exploit to $T_{e}$, the maximum layers of recursion can be written as $l=\max (\{|q|: q$ is an attack sequence satisfying $k 0 d(q, \phi)<k+1\})$. Solving 
the recurrence formula, we have that $t=|C| \cdot\left|E_{1}\right|^{2} \cdot\left|E_{0}\right|^{l}$. Therefore, the complexity is polynomial in the size of the zero day attack graph if $k$ is a constant.

\subsection{Computing $k$-Zero Day Safety as Shortest Paths in a DAG}

Although it is intractable to compute $k$ for arbitrary zero day attack graphs, efficient solutions may exist for those satisfying special properties. We now show such a case where the problem can be reduced to that of finding shortest paths in a directed acyclic graph (DAG). Roughly speaking, we make two assumptions: First, most exploits will only require one condition on the remote host (e.g., when a host is only used as a stepping stone, the condition could be a user privilege on that host); second, zero day exploits will be distinct unless they are on the same or adjacent hosts. Next, we informally describe our method while leaving the detailed algorithm and complexity analysis to Appendix C.

The first assumption implies that we can derive a logical proposition (as in Procedure $k 0 d \_B w d$ ) separately for each host. In the resultant DNF, each conjunctive clause will include at most one condition involving a remote host, which means the asset can be expressed as a disjunction of conditions (without considering exploits). We can thus repeat the same reasoning by regarding each such condition as an asset on the involved remote host. Since the relationships between all conditions are now disjunctive, we can regard each condition as the vertex of a DAG (recall that cycles will be avoided) with their disjunctive relationships as edges, and exploits in the same conjunctive clause as edge weights.

In the weighted DAG, determining the value of $k$ amounts to finding the shortest path along which the function $k 0 d($.$) applied to all zero day exploits$ will yield the minimum value. During a backward search, we keep two parts of a distance for each edge: For those zero day exploits that may later be related to others through $\equiv_{v}$, we keep them in a set since the function $k 0 d($.$) can not yet$ be applied; for other exploits, we only keep the result value of applying $k 0 d($.$) .$ The second assumption above essentially ensures that the first part of the edge distance will not grow quickly. The shortest distance can then be obtained using a standard algorithm [9], taking polynomial time (more precisely, the complexity is shown to be $|H|^{4} \cdot\left|E_{0}\right|$ in Appendix C).

\section{Discussions}

In this section, we demonstrate the power of our metric through an example application, network hardening, and discuss issues in instantiating the model.

Network Hardening Using the Metric Based on the proposed metric, network hardening can be defined as making a network $k$-zero day safe for a larger $k$. 
Such a concept generalizes the existing qualitative approach in [46], which essentially achieves $k>0$. Moreover, the metric immediately imply a collection of hardening options. To see this, we first unfold $k$ based on the model:

$$
\begin{aligned}
k= & k 0 d(A)=\sum_{a \in A}(k 0 d(a) \cdot v(a)) / \sum_{a \in A} v(a) \\
k 0 d(a)= & \min \left(\left\{k 0 d\left(q \cap E_{0}, \phi\right): q \in \operatorname{seq}(a)\right\}\right) \\
k 0 d\left(q \cap E_{0}, \phi^{\prime}\right)= & \max \left(\left\{|F|: F \subseteq q \cap E_{0},\left(\forall e_{1}, e_{2} \in F\right)\left(e_{1} \not \equiv_{v} e_{2}\right)\right\}\right) \\
\operatorname{seq}(a)= & \left\{e_{1}, e_{2}, \ldots, e_{j}: a \in \operatorname{post}\left(e_{j}\right),\right. \\
& \left.(\forall i \in[1, j])\left(\forall c \in \operatorname{pre}\left(e_{i}\right)\right)\left(c \in C_{I}\right) \vee\left(\exists x \in[1, i-1] c \in \operatorname{post}\left(e_{x}\right)\right)\right\}
\end{aligned}
$$

Therefore, $k$ can be increased by:

- Increasing services' diversity to have more distinct exploits in Equation (3).

- Strengthening isolation techniques for a similar effect as above.

- Disabling initial conditions (e.g., removing a service or a connection) in $C_{I}$ to yield longer attack sequences in above line (5) (part of Equation (4)).

- Enforcing more strict access control policies to lessen the risk of insider attacks or user mistakes (thus removing conditions from $C_{I}$ in line (5)).

- Protecting assets with backups (conjunction of conditions) and detection efforts (negation of conditions) to yield a longer sequence in Equation (4).

- Introducing more security services to regulate accesses to remote services for a longer sequence in Equation (4).

- Patching known vulnerabilities such that less shortcuts for bypassing zero day exploits yield a longer sequence in Equation (4).

- Prioritizing the above options based on the asset values in Equation (1) and shortest attack sequences in Equation (2).

Although the above hardening options are nothing new, their effectiveness is now quantified in a simple, intuitive way. The cost of network hardening can be easily justified, not based upon speculation or good will, but with a larger $k$.

Instantiating the Model Since the proposed metric and algorithms are based on an abstract model of networks, how to instantiate the model for given networks is an equally important (and admittedly difficult) issue. We now address several key aspects of the issue while leaving more research to future work.

- While instantiating the model, an uncertain situation can be dealt with by either taking a conservative assumption under which the metric yields a lower $k$ (e.g., any host should be included unless it is believed to be absolutely immune from zero day attacks) or by taking a probabilistic approach (e.g., we have discussed how associating a probability to relation $\equiv_{v}$ can help to model the degree of similarity in vulnerabilities and strength of isolation). Our future work will further explore such probabilistic approaches. 
- An extremely conservative assumption may yield a trivial result (e.g., no network is 1-zero day safe, if insider attacks are considered possible on every host). While such an assumption may be the safest, it is also the least helpful in terms of improving the security since nothing would be helpful.

- The remote services and network connectivity must be identified by examining hosts' configuration. A network scanning is insufficient since it will not reveal services or connectivity currently disabled by security services (e.g., ssh behind iptables in Figure 1). The model is thus more concerned about the existence, instead of the current reachability, of a service or host.

- A zero day attack graph cannot be obtained by injecting zero day exploits into an existing attack graph of known vulnerabilities. The reason is that some unreachable exploits may be discarded in generating an attack graph of known vulnerabilities [1], whereas such exploits may indeed serve as shortcuts for bypassing zero day exploits in a zero day attack graph.

- The model itself does not provide a means for determining which conditions are likely to be subject to insider attacks or user mistakes, which should be determined based on knowledge about access control polices (which users are allowed to do what on which hosts) and how trustworthy each user is.

\section{Related Work}

Standardization efforts on vulnerability assessment include the Common Vulnerability Scoring System (CVSS) [26,30] which measures vulnerabilities in isolation. The NIST's efforts on standardizing security metrics are also given in [27] and more recently in [43]. The research on security metrics has attracted much attention lately $[20,15,2,17]$. Earlier work include the intuitive properties of a security metric and a metric in terms of time and efforts based on a Markov model [7, 8,31]. More recently, an attack resistance metric based on attack graph is proposed in $[48,47]$ and another approach derives probabilistic scores for a network by combining CVSS scores $[45,13]$. The minimum efforts required for executing each exploit is used as a metric in [3]. Another approach measures the relative risk using the least set of initial conditions under which an attack is possible [35]. The cost of network hardening is quantified in $[29,46]$. The use of failure-time analysis for developing risk management metrics is discussed in [41]. A mean time-to-compromise metric is proposed based on the predator state-space model (SSM) used in the biological sciences in [22]. Security metrics are also developed for specific applications, such as the information-theoretic metrics for measuring the effectiveness of IDSs [21, 14], and for specific threats, such as botnets [16] and traffic analysis [28].

Relevant work exist in other areas. Design principles are proposed for developing metrics of trust [38,39], which we found useful in our work. A metric 
for measuring the trust in an identity established through overlapping chains of certificates is proposed in [4]. Security is considered as another risk that needs to be managed alongside all business risks [12]. A series of work on attack surface measures how likely a software is vulnerable to attacks based on the degree of exposure [18, 32-34,23]. Our work borrows from attack surface the idea of focusing on interfaces, instead of internal details, of a system. However, we apply the idea to a network of computer systems, instead of a single software system. Parallel to the study of security metrics, fault tolerance algorithms rely on replication and diversity to improve the availability of services $[5,6]$. Our metric provides a means for measuring the effectiveness of such algorithms in networks. Finally, our work is partially inspired by the well known data privacy metric $k$-anonymity [37] which measures the amount of privacy using an integer regardless of specific application semantic.

To generate attack graphs, topological vulnerability analysis enumerates potential sequences of exploits of known vulnerabilities [7, 11,31, 36, 49, 44]. Based on whether a search starts from the initial state or the final state, such an analysis can be forward [36,44] or backward [40,42]. In our study, we adopt the graph-based representation of attack graphs proposed in [1]. To the best of our knowledge, only limited work exist on measuring the effect of zero day attacks on networks. An empirical study of the total number of zero day vulnerabilities available on a single day is given based on existing data [25], and an effort on ordering different applications by the seriousness of consequences of having one a single zero day vulnerability (which is parallel to our work) [19].

\section{Conclusion}

We have proposed $k$-zero day safety as a novel security metric for measuring the relative security of networks against potential zero day attacks. In doing so, we have transformed the unmeasureability of unknown vulnerabilities from a commonly perceived obstacle to an opportunity for security metrics. While the general problem of computing the metric is intractable, we have demonstrated that practical security issues can be formulated and solved in polynomial time. For future work, we shall elaborate on probabilistic modeling approaches and the algorithms for hardening networks based on the metric; we shall also integrate the proposed algorithms into existing attack graph-based security tools so to validate their real world effectiveness. The proposed metric leads to many other interesting future directions, such as statistical modeling of network security using the metric, extensions to the metric with relaxed assumptions about zero day vulnerabilities, and applying the metric to special applications such as measuring the risk of worms and botnets. 


\section{References}

1. P. Ammann, D. Wijesekera, and S. Kaushik. Scalable, graph-based network vulnerability analysis. In Proceedings of the 9th ACM Conference on Computer and Communications Security (CCS'02), 2002.

2. A. C. S. Associates. Workshop on. In Information Security System Scoring and Ranking, 2001.

3. D. Balzarotti, M. Monga, and S. Sicari. Assessing the risk of using vulnerable components. In Proceedings of the 1st Workshop on Quality of Protection, 2005.

4. T. Beth, M. Borcherding, and B. Klein. Valuation of trust in open networks. In Proceedings of the Third European Symposium on Research in Computer Security (ESORICS'94), pages 3-18, 1994.

5. M. Castro and B. Liskov. Practical byzantine fault tolerance and proactive recovery. ACM Trans. Comput. Syst., 20(4):398-461, 2002.

6. B.-G. Chun, P. Maniatis, and S. Shenker. Diverse replication for single-machine byzantinefault tolerance. In ATC'08: USENIX 2008 Annual Technical Conference on Annual Technical Conference, pages 287-292, Berkeley, CA, USA, 2008. USENIX Association.

7. M. Dacier. Towards quantitative evaluation of computer security. Ph.D. Thesis, Institut National Polytechnique de Toulouse, 1994.

8. M. Dacier, Y. Deswarte, and M. Kaaniche. Quantitative assessment of operational security: Models and tools. Technical Report 96493, 1996.

9. E. W. Dijkstra. A note on two problems in connection with graphs. Numerische Mathematik, 1:269271, 1959.

10. J. Doob. Measure Theory. Springer-Verlag, 1994.

11. D. Farmer and E. Spafford. The COPS security checker system. In USENIX Summer, pages 165-170, 1990.

12. S. N. Foley. Security risk management using internal controls. In WISG '09: Proceedings of the first ACM workshop on Information security governance, pages 59-64, New York, NY, USA, 2009. ACM.

13. M. Frigault, L. Wang, A. Singhal, and S. Jajodia. Measuring network security using dynamic bayesian network. In Proceedings of ACM workshop on Quality of protection (QoP'08), 2008.

14. G. Gu, P. Fogla, D. Dagon, W. Lee, and B. Skoric. Towards an information-theoretic framework for analyzing intrusion detection systems. In European Symposium on Research in Computer Security, pages 527-546, 2006.

15. D. Herrmann. Complete Guide to Security and Privacy Metrics. Auerbach Publications, 2007.

16. T. Holz, C. Gorecki, K. Rieck, and F. C. Freiling. Measuring and detecting fast-flux service networks. In Annual Network and Distributed System Security Symposium, 2008.

17. K. Hoo. Metrics of network security. White Paper, 2004.

18. M. Howard, J. Pincus, and J. Wing. Measuring relative attack surfaces. In Workshop on Advanced Developments in Software and Systems Security, 2003.

19. K. Ingols, M. Chu, R. Lippmann, S. Webster, and S. Boyer. Modeling modern network attacks and countermeasures using attack graphs. In ACSAC '09: Proceedings of the 2009 Annual Computer Security Applications Conference, pages 117-126, Washington, DC, USA, 2009. IEEE Computer Society.

20. A. Jaquith. Security Merics: Replacing Fear Uncertainity and Doubt. Addison Wesley, 2007.

21. W. Lee and D. Xiang. Information-theoretic measures for anomaly detection. In Proceedings of the 2001 IEEE Symposium on Security and Privacy, page 130, Washington, DC, USA, 2001. IEEE Computer Society. 
22. D. J. Leversage and E. J. Byres. Estimating a system's mean time-to-compromise. IEEE Security and Privacy, 6(1):52-60, 2008.

23. K. Manadhata, J. Wing, M. Flynn, and M. McQueen. Measuring the attack surfaces of two ftp daemons. In ACM workshop on Quality of Protection, 2006.

24. J. McHugh. Quality of protection: Measuring the unmeasurable? In Proceedings of the 2nd ACM workshop on Quality of protection (QoP'06), pages 1-2, 2006.

25. M. McQueen, T. McQueen, W. Boyer, and M. Chaffin. Empirical estimates and observations of Oday vulnerabilities. Hawaii International Conference on System Sciences, 0:1-12, 2009.

26. P. Mell, K. Scarfone, and S. Romanosky. Common vulnerability scoring system. IEEE Security \& Privacy Magazine, 4(6):85-89, 2006.

27. National Institute of Standards and Technology. Technology assessment: Methods for measuring the level of computer security. NIST Special Publication 500-133, 1985.

28. R. E. Newman, I. S. Moskowitz, P. F. Syverson, and A. Serjantov. Metrics for trafic analysis prevention. In Privacy Enhancing Technologies, pages 48-65, 2003.

29. S. Noel, S. Jajodia, B. O'Berry, and M. Jacobs. Efficient minimum-cost network hardening via exploit dependency grpahs. In Proceedings of the 19th Annual Computer Security Applications Conference (ACSAC'03), 2003.

30. National vulnerability database. available at: http://www.nvd.org, May 9, 2008.

31. R. Ortalo, Y. Deswarte, and M. Kaaniche. Experimenting with quantitative evaluation tools for monitoring operational security. IEEE Trans. Software Eng., 25(5):633-650, 1999.

32. J. W. P. Manadhata. Measuring a system's attack surface. Technical Report CMU-CS-04$102,2004$.

33. J. W. P. Manadhata. An attack surface metric. Technical Report CMU-CS-05-155, 2005.

34. J. W. P. Manadhata. An attack surface metric. In First Workshop on Security Metrics (MetriCon), 2006.

35. J. Pamula, S. Jajodia, P. Ammann, and V. Swarup. A weakest-adversary security metric for network configuration security analysis. In Proceedings of the 2nd ACM workshop on Quality of protection, pages 31-38, New York, NY, USA, 2006. ACM Press.

36. C. Phillips and L. Swiler. A graph-based system for network-vulnerability analysis. In Proceedings of the New Security Paradigms Workshop (NSPW'98), 1998.

37. P.Samarati. Protecting respondents' identities in microdata release. In IEEE Transactions on Knowledge and Data Engineering (TKDE), pages 1010-1027, 2001.

38. M. Reiter and S. Stubblebine. Authentication metric analysis and design. ACM Transactions on Information and System Security, 2(2):138-158, 51999.

39. M. K. Reiter and S. G. Stubblebine. Toward acceptable metrics of authentication. In Proceedings of the 1997 IEEE Symposium on Security and Privacy, page 10, Washington, DC, USA, 1997. IEEE Computer Society.

40. R. Ritchey and P. Ammann. Using model checking to analyze network vulnerabilities. In Proceedings of the 2000 IEEE Symposium on Security and Privacy, pages 156-165, 2000.

41. J. J. C. H. Ryan and D. J. Ryan. Performance metrics for information security risk management. IEEE Security and Privacy, 6(5):38-44, 2008.

42. O. Sheyner, J. Haines, S. Jha, R. Lippmann, and J. Wing. Automated generation and analysis of attack graphs. In Proceedings of the 2002 IEEE Symposium on Security and Privacy, 2002.

43. M. Swanson, N. Bartol, J. Sabato, J. Hash, and L. Graffo. Security metrics guide for information technology systems. NIST Special Publication 800-55, 2003.

44. L. Swiler, C. Phillips, D. Ellis, and S. Chakerian. Computer attack graph generation tool. In Proceedings of the DARPA Information Survivability Conference \& Exposition II (DISCEX'01), 2001. 
45. L. Wang, T. Islam, T. Long, A. Singhal, and S. Jajodia. An attack graph-based probabilistic security metric. In Proceedings of The 22nd Annual IFIP WG 11.3 Working Conference on Data and Applications Security (DBSec'08), 2008.

46. L. Wang, S. Noel, and S. Jajodia. Minimum-cost network hardening using attack graphs. Computer Communications, 29(18):3812-3824, 112006.

47. L. Wang, A. Singhal, and S. Jajodia. Measuring network security using attack graphs. In Proceedings of the 3rd ACM workshop on Quality of protection (QoP'07), New York, NY, USA, 2007. ACM Press.

48. L. Wang, A. Singhal, and S. Jajodia. Measuring the overall security of network configurations using attack graphs. In Proceedings of 21th IFIP WG 11.3 Working Conference on Data and Applications Security (DBSec'07), 2007.

49. D. Zerkle and K. Levitt. Netkuang - a multi-host configuration vulnerability checker. In Proceedings of the 6th USENIX Unix Security Symposium (USENIX'96), 1996.

\section{Appendix A: Proof of Theorem 1}

Proof: This is to prove, for all $F, F^{\prime}, F^{\prime \prime} \subseteq E_{0}$, the following hold [10].

1. $k 0 d\left(F, F^{\prime}\right)=0$ iff $F=F^{\prime}$ : This is straightforward since $k 0 d\left(F, F^{\prime}\right)=0$ iff $F \triangle F^{\prime}=\phi$, and the latter is equivalent to $F=F^{\prime}$.

2. $k 0 d\left(F, F^{\prime}\right)=k 0 d\left(F^{\prime}, F\right)$ : This property is satisfied by the symmetric difference.

3. $k 0 d\left(F, F^{\prime}\right)+k 0 d\left(F^{\prime}, F^{\prime \prime}\right) \geq k 0 d\left(F, F^{\prime \prime}\right)$ : Denote by $\operatorname{tmp}(G)$ the function $\max \left(\left\{\left|G^{\prime}\right|: G^{\prime} \subseteq G, \forall e_{1}, e_{2} \in G^{\prime}\left(e_{1} \not \equiv v e_{2}\right)\right\}\right)$. First, the symmetric difference satisfies the triangle inclusion relation $F \triangle F^{\prime \prime} \subset\left(F \triangle F^{\prime}\right) \cup$ $\left(F^{\prime} \triangle F^{\prime \prime}\right)$ [10]. So, $\operatorname{tmp}\left(\left(F \triangle F^{\prime}\right) \cup\left(F^{\prime} \triangle F^{\prime \prime}\right)\right) \geq \operatorname{tmp}\left(F \triangle F^{\prime \prime}\right)$ holds. Next, we only need to show $\operatorname{tmp}\left(F \triangle F^{\prime}\right)+\operatorname{tmp}\left(F^{\prime} \triangle F^{\prime \prime}\right) \geq \operatorname{tmp}\left(\left(F \triangle F^{\prime}\right) \cup\right.$ $\left.\left(F^{\prime} \triangle F^{\prime \prime}\right)\right)$ is true. It suffices to show the function $\operatorname{tmp}($.$) to be subadditive,$ that is, $\operatorname{tmp}(G)+\operatorname{tmp}\left(G^{\prime}\right) \geq \operatorname{tmp}\left(G \cup G^{\prime}\right)$ holds for any $G, G^{\prime} \subseteq E_{0}$. This follows from the fact that if the relation $e \equiv_{v} e^{\prime}$ holds for any $e, e^{\prime} \in G$ (or $e, e^{\prime} \in G^{\prime}$ ), it also holds in $G \cup G^{\prime}$ (the converse is not necessarily true).

\section{Appendix B: Proof of Theorem 2}

Proof: First, the problem is NP, since whether a given sequence of exploits $q$ satisfies $q \in \operatorname{seq}(a) \wedge k 0 d\left(q \cap E_{0}, \phi\right)=k$ can be easily determined in polynomial time in the size of the zero day attack graph.

Next, we reduce the NP-hard problem of finding the minimum attack (that is, an attack sequence with the minimum number of exploits) in attack graph [1, 42] to the current problem. First of all, the reduction is not trivial. More precisely, the reduction cannot be trivially achieved by simply replacing each known 
exploit with a zero day exploit in a given attack graph of know exploits, because, unlike the former, the latter has a fixed number of hard-coded pre- and post-conditions that may prevent them from fitting in the position of a known exploit.

We construct a zero day attack graph $G^{\prime}$ by injecting a zero day exploit before each known exploit. Specifically, first let $G^{\prime}=G$. Then, for each known exploit $e$ of a service $s$ from a source host $h_{1}$ to a different destination host $h_{2}$, we inject a zero day exploit $e^{\prime}$ with the post-conditions $\left\{\left\langle s, h_{2}\right\rangle, p_{\text {useless }}\right\}$ where $p_{\text {userless }}$ is a privilege designed not to be the pre-condition of any exploit ( $e^{\prime}$ can be interpreted as exploiting a vulnerability in a security service, such as a personal firewall, that blocks accesses to the service $s$ on $h_{2}$ from $h_{1}$ ). We then have the following two facts. First, executing $e$ requires $e^{\prime}$ to be executed first; conversely, if $e^{\prime}$ needs to be executed, then the only reason must be to satisfy the condition $\left\langle s, h_{2}\right\rangle$ and consequently execute $e$. That is, any attack sequence in $G^{\prime}$ will include either both $e$ and $e^{\prime}$, or none of them. Second, among the three conditions in pre $\left(e^{\prime}\right)=\left\{\left\langle s^{\prime}, h_{2}\right\rangle,\left\langle h_{1}, h_{2}\right\rangle,\left\langle p_{\text {least }}, h_{1}\right\rangle\right\}$, the first is an initial condition and the last two are also members of pre $(e)$. Therefore, the injection of $e^{\prime}$ does not change the logical structure of the attack graph (more precisely, $G$ and $G^{\prime}$ are isomorphic if we regard $e$ and $e^{\prime}$ as a single exploit and ignore the initial condition $\left.\left\langle s^{\prime}, h_{2}\right\rangle\right)$.

Next, for each known exploit $e$ involving the same source and destination host $h$, we replace $e$ with a zero day exploit $e^{\prime}$ and a known exploit $e^{\prime \prime}$ satisfying that $\operatorname{post}\left(e^{\prime \prime}\right)=\operatorname{post}(e), \operatorname{pre}\left(e^{\prime \prime}\right)=\operatorname{pre}(e) \backslash\{\langle p, h\rangle\} \cup\left\{\left\langle p^{\prime}, h\right\rangle\right\}$ where $\langle p, h\rangle \in$ $\operatorname{pre}(e)$ and $\left\{\left\langle p^{\prime}, h\right\rangle\right\}$ are two privileges. We also let $\operatorname{post}\left(e^{\prime}\right)=\left\{\left\langle p^{\prime}, h\right\rangle\right\}$, and design the relation $\equiv_{v}$ in such a way that $e^{\prime}$ is not related to any other zero day exploits in $h$ through $\equiv_{v}$. We then have two similar facts as above. First, any attack sequence in $G^{\prime}$ will include either both $e$ and $e^{\prime}$, or none of them. Second, the injection of $e^{\prime}$ does not change the logical structure of the attack graph.

Based on the above construction, given any asset $a$, for any attack sequence $q^{\prime} \in \operatorname{seq}(a)$ in $G^{\prime}$, the known exploits in $q$ also form an attack sequence $q \in$ $\operatorname{seq}(a)$ in $G$ (note that $a$ will always be the post-condition of known exploits due to our construction). Moreover, if we design $\equiv_{v}$ in such a way that no two zero day exploits are related by $\equiv_{v}$, then we have $|q|=k 0 d\left(q^{\prime} \cap E_{0}, \phi\right)$. Therefore, for any non-negative integer $k$, finding $q^{\prime}$ in $G^{\prime}$ to minimize $k 0 d\left(q^{\prime} \cap E_{0}, \phi\right)$ will immediately yield $q$ in $G$ that also minimizes $|q|$, and the latter is essentially the minimum attack problem. This shows the former to be an NP-hard problem and concludes the proof. 


\section{Appendix C: A Procedure for Computing $k$-Zero Day Safety as Shortest Paths in a DAG}

In Figure 5, Procedure $k 0 d$ Shortest provides a more precise description of the method informally discussed in Section 3.3. Sub_Procedure k0d_Graph is used to build a DAG based on a given zero day attack graph and asset. First, the sub-procedure derives a logical proposition of the asset in terms of exploits and conditions using the same statements as in Procedure k0d_Backward (line 17), but stops whenever the DNF of the logic proposition includes at most one condition in each conjunctive clause (lines 18-19). The sub-procedure then adds each such conjunctive clause to the result DAG (line 20) by regarding each condition as a vertex pointed to by the asset (lines 21-22), and the set of exploits in the same conjunctive clause as the edge weight (line 23). The sub-procedure then recursively expands on each such condition (line 24). If a conjunctive clause does not include a condition (meaning that only initial conditions are required), a dummy vertex is added to represent the collection of deleted initial conditions (line 26-27).

The main procedure then imitates a standard algorithm for finding the shortest path in a DAG [9]. More specifically, the vertices are processed based on a topological sort (line 3); and the distance of the source vertex is initialized as 0 while that of other vertices as infinity (line 4); upon processing a vertex (line 5-6), each of its neighbors (line 7) will be updated with potentially shorter distances via the current vertex (lines 9-14). Finally, the main procedure returns the minimum shortest distance from the asset to a dummy vertex (representing initial conditions) as the result $k$.

The following modifications to the standard shortest distance algorithm are designed to take into account zero day exploits related by $\equiv_{v}$. First, instead of a single number, each distance is now a set of pairs $\langle x, y\rangle$ where $x$ denotes the result of applying $k 0 d($.$) to exploits that will not later be related to others by \equiv_{v}$, whereas $y$ denotes the set of zero day exploits that may later be related to others. More than one pairs may be necessary for a distance since they are incomparable due to the latter. Second, the reachable edges are collected in order to determine whether an exploit may later be related to others by $\equiv_{v}$ (line 8). Third, instead of simply calculating the minimum distance, both parts of each distance pair must be computed based on the distance of current vertex and the edge weight (line 10-11). The new distance pair will then immediately be added (line 12). Finally, after all distance pairs are added, the set of distance pairs is examined again to remove those that cannot be the minimum distance even when considering the effect of relation $\equiv_{v}$ (line 13-14). 


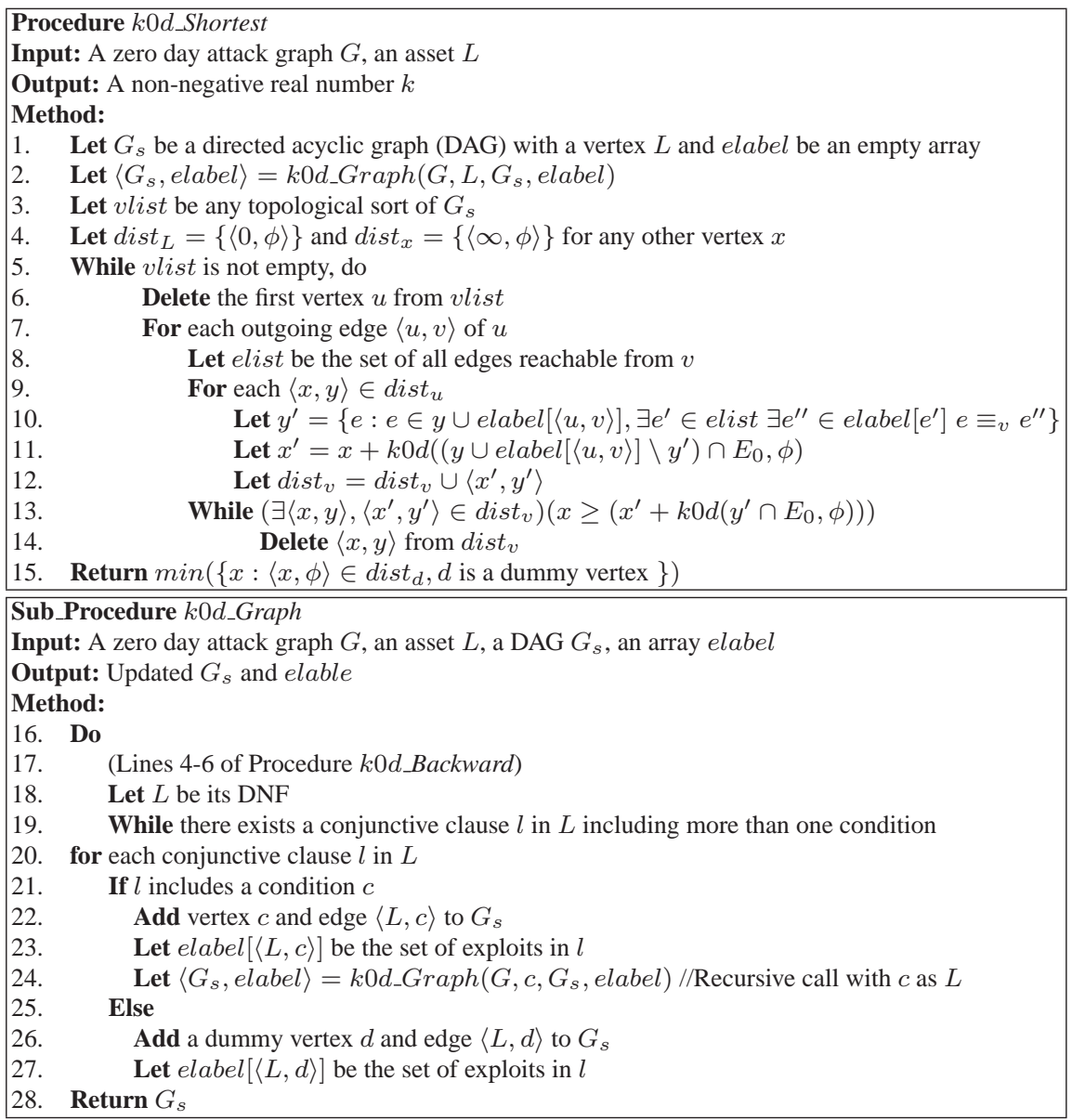

Fig. 5. Computing $k$-Zero Day Safety as Shortest Paths in a DAG

Example 4. For our running example, Figure 6 illustrates the execution of Procedure $k 0 d \_$Shortest. Each edge is labeled with the edge weight elabel and each vertex with the distance dist.

Complexity The complexity of the procedure will depend on how well the aforementioned assumptions hold on a given zero day attack graph. First, the complexity of Sub-Procedure k0d_Graph is exponential in the number of exploits and conditions involved in the loop at lines 16-19. Therefore, if the first assumption perfectly holds, this loop will always terminate after processing a single host. If we regard the number of exploits and conditions on each host as a constant, then the complexity of the sub-procedure will be linear in the number of hosts (that is, a constant time is required for deriving and processing $L$ for 


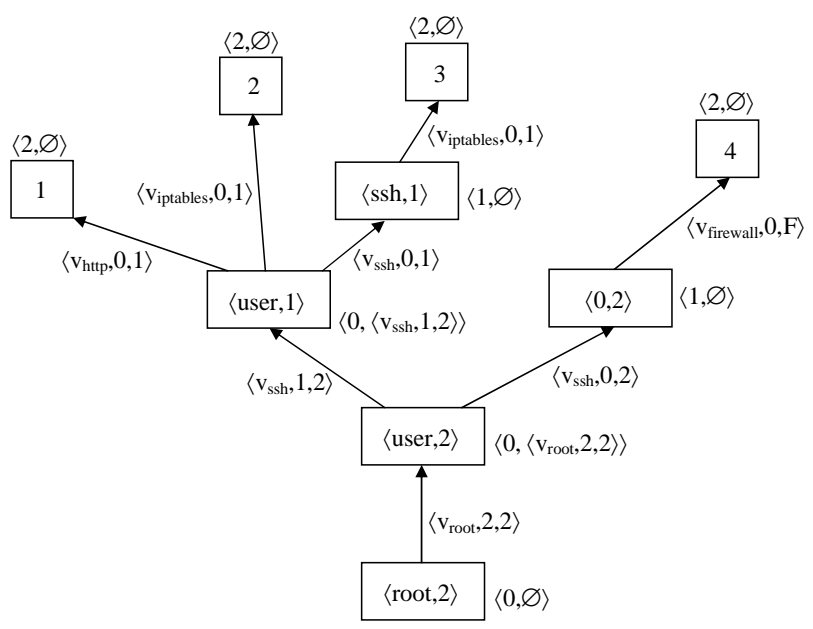

Fig. 6. An Example of Executing Procedure $k 0 d \_S h o r t e s t$

each host). Second, the complexity of the main procedure depends on the size of the distance of each vertex. If the second assumption holds perfectly such that each distance has a negligible size, then the complexity of the main procedure will be dominated by processing the reachable edges in elist and their labels elabel (line 10). Since each edge in $G_{s}$ is visited exactly once by the main loop and the size of elist is linear in the number of such edges, the processing of elist takes quadratic time in the number of edges in $G_{s}$, which is roughly $O\left(|H|^{4}\right)$ (by the first assumption, each host corresponds to a constant number of vertices in $G_{s}$ ). Finally, multiplying this by the size of elabel, we have the complexity $|H|^{4} \cdot\left|E_{0}\right|$. 